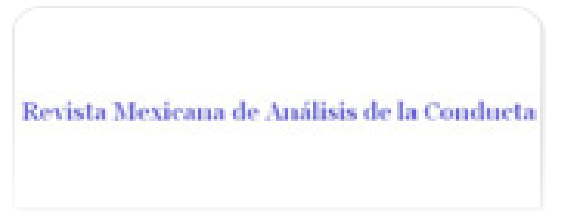

Revista Mexicana de Análisis de la Conducta ISSN: 0185-4534

editora@rmac-mx.org

Sociedad Mexicana de Análisis de la Conducta México

Rodríguez Pérez, María Elena

Adquisición de una discriminación condicional bajo diferentes historias de entrenamiento de correspondencia

Revista Mexicana de Análisis de la Conducta, vol. 33, núm. 2, 2007, pp. 183-203

Sociedad Mexicana de Análisis de la Conducta

Guadalajara, México

Disponible en: http://www.redalyc.org/articulo.oa?id=59333205

Cómo citar el artículo

- Número completo

- Más información del artículo

Página de la revista en redalyc.org

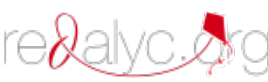

Sistema de Información Científica

Red de Revistas Científicas de América Latina, el Caribe, España y Portugal Proyecto académico sin fines de lucro, desarrollado bajo la iniciativa de acceso abierto 


\title{
ADQUISICIÓN DE UNA DISCRIMINACIÓN CONDICIONAL BAJO DIFERENTES HISTORIAS DE ENTRENAMIENTO DE CORRESPONDENCIA
}

\author{
ACQUISITION OF A CONDITIONAL DISCRIMINATION UNDER \\ DIFFERENT CORRESPONDENCE TRAINING HISTORIES
}

MARÍA ELENA RODRÍGUEZ PÉREZ

UNIVERSIDAD DE GUADALAJARA

\begin{abstract}
RESUMEN
El entrenamiento de correspondencia es un procedimiento cuyo propósito es fortalecer, desarrollar, eliminar o debilitar cierta conducta mediante la verbalización de lo que se va a hacer o la descripción de lo que se hizo. En el presente estudio, se modificó una tarea de igualación de la muestra de segundo orden para someter a participantes de dos poblaciones estudiantiles distintas a diferentes historias de entrenamiento de correspondencia decir-hacer, hacer-describir y decir-describir para analizar los efectos de este entrenamiento sobre la adquisición y generalidad de la discriminación condicional. El entrenamiento de correspondencia interfirió con la adquisición de la igualación ya que los participantes que aprendieron la discriminación condicional lo hicieron bajo la condición de prueba de transferencia. Estos resultados parecen sugerir que el establecimiento de correspondencias entre lo que se dice que se va a hacer, lo que se hace y lo que se describe que se hizo puede estar asociado a la intervención funcional de la retroalimentación, es decir, a la manera en que la retroalimentación favorece que un factor o la relación entre dos factores de la tarea de igualación logre aparecer con un peso relativo mayor que los otros elementos de las relaciones contingenciales entrenadas.

Palabras claves: Igualación de la muestra, entrenamiento de correspondencia, discriminación condicional.
\end{abstract}

1. Dirigir correspondencia a: Centro de Estudios e Investigaciones en Comportamiento, Francisco de Quevedo 180. Colonia Arcos Vallarta. Guadalajara, Jalisco, México. CP. 44130. Correo electrónico: rpm08428@cucba.udg.mx. 


\begin{abstract}
Correspondence training is a procedure used to promote, eliminate, weaken or increment a target behavior through the verbalization of future or past correspondent behaviors. In this experiment, a second-order matching-to-sample task was modified to train subjects from two different school populations into different correspondence training histories (saying-doing, doing-describing and saying-describing) in order to analyze the effects of correspondence training on acquisition and generalization of the conditional discrimination. Correspondence training interfered with the acquisition of matching to sample and those subjects who learnt the conditional discrimination did it under the transfer test condition. These results suggest that the establishment of correspondence among what a subject says he is going to do, what he does and what he describes that did may be associated to functional intervention of feedback, that is, to the way feedback promotes that a factor or the relation of two factors of the matching-to-sample task appears with a greater relative weight than the other elements of the contingencial relations trained.

Key words: Matching-to-sample, correspondence training, conditional
\end{abstract} discrimination

El estudio de las interacciones entre los elementos no verbales y verbales de una tarea, referidas como ejemplos de "regulación verbal", "control verbal", "mediación verbal" o "actividad voluntaria", parte del supuesto de que la actividad verbal juega un papel directivo de la correspondiente actividad motora (Bentall, Lowe y Beasty, 1985; Birch, 1966; Higa, Tharp y Calkins, 1978; Israel y O'Leary, 1973; Lovaas, 1961; Meacham, 1979; Meichenbaum y Goodman, 1969; Rogers-Warren y Baer, 1976). Sin embargo, el trabajo de Brodsky (1967) puso en duda el presupuesto de una relación directa e inmediata entre conducta verbal y no verbal. Dicho autor no pudo aumentar la conducta social de una paciente con retardo mental después de reforzar sus intenciones explícitas de emitir tal conducta. Es así como nació el campo de entrenamiento de correspondencia cuyo propósito es investigar los factores y variables de las cuales depende que se establezca una relación entre la conducta verbal y no verbal en lugar de presuponerla (Israel, 1978).

En uno de los primeros estudios, Risley y Hart (1968) utilizaron un procedimiento en que se reforzó que niños preescolares describieran lo que habían hecho durante el recreo. Posteriormente, se dio comida sólo a los niños que decían que habían realizado una de las dos tareas objetivo (jugar con bloques o pintar), independientemente de lo que hubieran hecho (reforzamiento del contenido). Los resultados mostraron que el reforzamiento del contenido hizo que todos los niños reportaran las tareas objetivo, pero no 
produjo un aumento en la tasa de ocurrencia de dichas actividades. Ensegui$\mathrm{da}$, solamente se les dio comida a quienes jugaron con bloques o pintaron y así lo reportaron (entrenamiento de correspondencia). El entrenamiento de correspondencia favoreció que sólo los niños que habían realizado una de las dos tareas objetivos reportaran haberlo hecho.

Posteriormente, Israel y Brown (1977) cuestionaron la pertinencia del entrenamiento con reforzamiento del contenido como fase anterior al entrenamiento de correspondencia ya que no encontraron diferencias en las ejecuciones de dos grupos de niños, uno sometido a reforzamiento de contenido y el otro no. Además, Israel (1978) distinguió dos estrategias diferentes de entrenamiento: (1) reforzamiento del reporte verbal de su conducta no verbal pasada con la esperanza de que esto produjera cambios en la futura conducta no verbal correspondiente; y (2) alentar a los individuos a usar sus propias conductas verbales para cambiar las conductas no verbales correspondientes. A la primera estrategia se le denominó entrenamiento de correspondencia hacer-decir y a la segunda entrenamiento de correspondencia decir-hacer. Este autor planteó la necesidad de estudiar a la correspondencia en relación al papel funcional de las verbalizaciones ya que Israel y O'Leary (1973) encontraron que un tipo de correspondencia no se generalizó al otro.

A decir de Lloyd (2002) el área de entrenamiento de correspondencia fue "abandonada" en 1992. Sin embargo, los procedimientos básicos de entrenamiento de correspondencia aún se siguen utilizando en los contextos donde se desea alterar uno de los aspectos, motores o verbales, de una conducta por la manipulación de su contraparte (Bevill, Gast, Maguire y Vail, 2001; Herruzo y Luciano,1994; Luciano, Herruzo y Barnes-Holmes, 2001; Luciano, Molina-Cobos y Gómez-Becerra, 2000; Morrison, Sainato, Benchaaban y Endo, 2002; Olsen-Woods, Miltenberger y Foreman, 1998; Paniagua, 1978, 1990 y 2004; Paniagua y Baer,1982 y 1988; Ralph, Hogan, Hill, Perkins, Ryan y Strong, 1998; Stokes, Cameron, Dorsey y Fleming, 2004). Las poblaciones que han participado en estos estudios han sido niños de preescolar, individuos con retardo mental, adolescentes y estudiantes universitarios. Herruzo y Luciano (1994) han señalado que el uso casi exclusivo de niños como sujetos experimentales no es fortuito. Detrás de los estudios de entrenamiento de correspondencia se encuentra la especulación de Luria (1964) de que durante el desarrollo temprano de la conducta verbal, ésta no controla naturalmente a la conducta no verbal, pero que dicho control puede establecerse a través de un proceso de enseñanza (Whitman, Scibak, Butler, Ritcher, y Johnston, 1982).

En años recientes, se ha considerado que el uso de tareas de igualación de la muestra puede ser una estrategia metodológica apropiada en el estudio del establecimiento de correspondencias entre el decir y el hacer (Lattal y Doepke, 2001; Lloyd, 2002; Ribes y Rodríguez, 2001; Rodríguez, 2000 
y 2002). En dichas tareas se presentan cuando menos tres estímulos: un estímulo muestra (EM) y dos estímulos de comparación (ECOs) (Cumming y Berryman, 1965). Como los procedimientos de igualación de la muestra permiten fomentar relaciones entre estímulos independientes de sus propiedades fisicoquímicas particulares, los requisitos de respuesta correcta pueden ser establecidos con gran precisión (Ribes, Moreno y Martínez, 1998). El dato de interés no es sólo la frecuencia de una respuesta sino también la precisión con la que el sujeto se ajusta al criterio de igualación que le impone la situación experimental. Así, las respuestas a los ECOs pueden ser retroalimentadas de acuerdo a una regla predeterminada que puede incluir relaciones arbitrarias (igualación simbólica) o relaciones directas que se usan en los contextos lingüísticos fuera del laboratorio como la de semejanza, diferencia o identidad (Ribes, 1990).

En la investigación con sujetos humanos, se han distinguido dos tipos de tareas de igualación de la muestra: de primer y segundo orden. La primera presenta un estímulo de muestra y un mínimo de dos estímulos de comparación, de entre los que el sujeto tiene que elegir aquel que guarda una correspondencia funcional con las propiedades del estímulo muestra (Ribes, Torres, Barrera y Ramírez, 1995). En una tarea de igualación de la muestra de segundo orden (Fujita, 1983) se presentan arreglos de estímulos distribuidos horizontalmente en hileras. Generalmente, la primera hilera incluye uno o dos estímulos (denominados de segundo orden o selectores) que muestran la relación que será retroalimentada positivamente. En la segunda hilera aparece por lo menos un estímulo de muestra a ser igualado con base en algún criterio. En la última hilera aparecen dos o más estímulos de comparación. El sujeto tiene que seleccionar uno ( $\mathrm{y}$ a veces más de uno) de entre los estímulos de comparación, de acuerdo con una relación apropiada con los estímulos de muestra que está prescrita por los estímulos de segundo orden. Estos estímulos pueden relacionarse de varias maneras, pero las relaciones más generales son las de identidad, diferencia, semejanza, inclusión, exclusión y orden (Ribes, 1990). Los criterios que caracterizan a cada relación pueden concebirse como un continuo cuyos polos opuestos son los casos de identidad (poseer todos los elementos iguales) y diferencia (no tener elementos comunes). Dentro de estos dos polos, pueden pensarse muchos casos de semejanza (poseer ciertos elementos comunes pero otros no). Por ejemplo, la igualación por semejanza en color tomaría como respuesta correcta aquel estímulo de comparación que compartiera el color del estímulo de muestra aunque ellos difieran en tamaño, forma, rotación, etc. Es importante hacer notar que en tareas de igualación de la muestra de primer orden, el sujeto depende de su ejecución y la retroalimentación de su ejecución para identificar el criterio de igualación que está operando en un momento dado del entrenamiento, mientras que en una tarea de igualación de la muestra de segundo 
orden, la relación en operación se ejemplifica además con los estímulos de segundo orden (Ribes, Torres y Ramírez, 1996).

Así, en un experimento previo, se modificó una tarea de igualación de la muestra de segundo orden para permitir el entrenamiento de correspondencia (Rodríguez, 2000). Cada ensayo contempló tres pantallas en donde el participante "dijo" cuál ECO elegiría durante la igualación, "hizo" la tarea y "describió" lo que hizo. Se definieron tres tipos de entrenamiento de correspondencia, decir-hacer (DH), hacer-describir (HD) y decir-describir (DD), dependiendo de la manipulación de las consecuencias. Con esta tarea, se entrenaron participantes adultos en algún tipo de correspondencia antes o después de un entrenamiento instrumental para evaluar el efecto del entrenamiento de correspondencia en la adquisición y generalidad de la discriminación condicional. Los resultados mostraron que el entrenamiento de correspondencia interfirió con la adquisición de la tarea en algunos participantes cuando se utilizó antes del entrenamiento instrumental. Sin embargo, cuando los participantes ya dominaban la ejecución correcta en la tarea de discriminación condicional, se establecieron los tres tipos de correspondencias decir-hacer, hacer-describir y decir-describir con sólo retroalimentar uno de ellos. A partir de estos hallazgos, en el presente estudio, se deseaba evaluar el efecto de diversas historias de entrenamiento de correspondencia $\mathrm{DH}, \mathrm{HD}$ y $\mathrm{DD}$ sobre la adquisición y generalidad de la discriminación condicional.

\section{METODO}

\section{Participantes}

Participaron 23 estudiantes de dos poblaciones distintas. Ocho hombres y ocho mujeres de entre 16 y 18 años de edad eran estudiantes de un bachillerato tecnológico. Seis hombres y una mujer de entre 18 y 22 años de edad eran estudiantes de ingeniería. Ninguno tenía experiencia en la tarea experimental. A cambio de su participación, los estudiantes de bachillerato acumularon horas de servicio social, mientras que los estudiantes de ingeniería recibieron créditos en un curso universitario.

\section{Diseño}

Los participantes se dividieron al azar para formar 3 grupos experimentales y 2 controles con participantes de ambas poblaciones. En los grupos experimentales, 4 participantes fueron de bachillerato y 1 de ingeniería mientras que los grupos controles se formaron por 2 participantes de bachillerato y 2 de ingeniería. 
El diseño contempló cuatro fases de entrenamiento. Durante las tres primeras fases, los grupos experimentales fueron expuestos a diferentes historias de entrenamiento de correspondencia (ver tabla 1). En este entrenamiento, la retroalimentación sólo tomó en cuenta que el participante fuera congruente en sus elecciones sin importar que dicha respuesta fuera correcta o no (ver el procedimiento para una descripción más detallada). Por ello, en la fase 4, se sometieron a un entrenamiento instrumental en donde se retroalimentó las elecciones correctas para evaluar el efecto de las diferentes historias de entrenamiento sobre la adquisición de la igualación.

El grupo control 1 se sometió, durante las tres primeras fases, a una tarea similar a la utilizada durante el entrenamiento de correspondencia pero retroalimentando las elecciones correctas para evaluar el efecto de dichas diferencias en la retroalimentación. El grupo control 2 se sometió a un entrenamiento instrumental durante todo el experimento.

Después de cada fase, todos los participantes se sometieron a pruebas de transferencia para evaluar el grado de generalidad de la discriminación condicional adquirida durante el entrenamiento (Varela y Quintana, 1995). La preprueba y postprueba fueron sesiones idénticas presentadas al inicio y final del experimento para evaluar el aprendizaje de la discriminación condicional de cada participante.

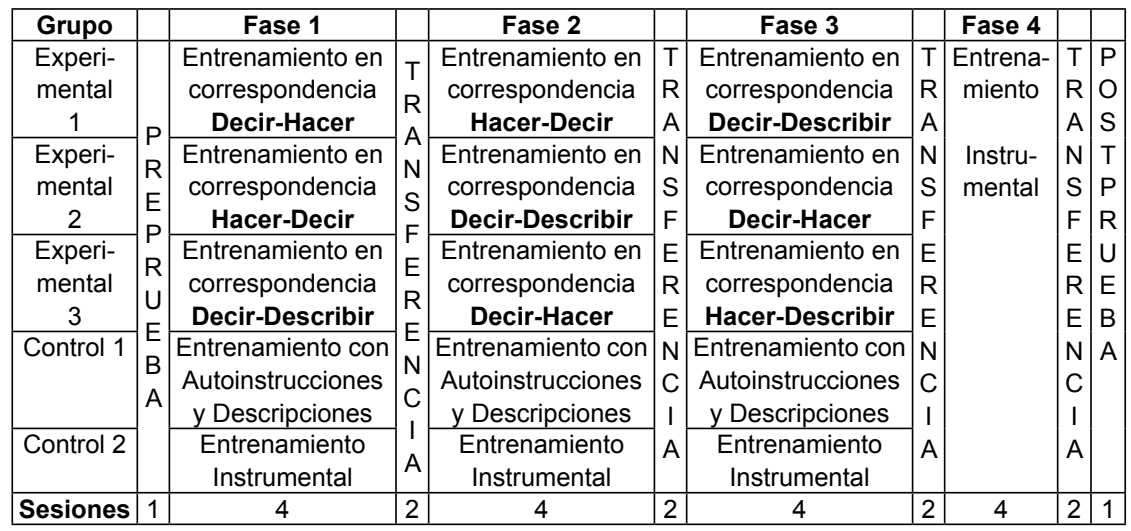

Tabla 1. Diseño para el experimento. 


\section{Procedimiento}

\section{Entrenamiento de correspondencia.}

La tarea de igualación de la muestra de segundo orden (ver figura 1) consistió de 36 ensayos que se presentaron en tres pantallas consecutivas: (1) autoinstrucciones, donde el participante hizo explícito cuál estímulo de comparación iba a elegir; (2) igualación, donde el participante resolvió la tarea de igualación; y (3) descripciones, donde el participante eligió un texto, de entre varias opciones, que mejor describiera lo que hizo durante la igualación.

En la pantalla de autoinstrucciones se describió al participante el arreglo de segundo orden por medio de un texto que aparecía en la parte superior de la pantalla. En la parte inferior, aparecía la pregunta "¿cuál de las figuras de abajo vas a seleccionar como respuesta correcta?" seguida de una serie de opciones a elegir con el ratón de la computadora.

En la pantalla de igualación, se utilizaron arreglos de estímulos distribuidos horizontalmente en tres hileras. En la primera hilera, se presentaron dos estímulos de segundo orden, en la segunda hilera apareció un estímulo de muestra y en la tercera hilera se tenían tres estímulos de comparación (ECO). Los estímulos fueron figuras geométricas cromadas diseñadas a partir de cinco formas y cinco colores distintos. Las formas y colores utilizados como estímulos de segundo orden fueron diferentes a los usados como estímulos muestra y de comparación. Cada ECO ejemplificaba una de las tres relaciones manejadas: (1) identidad, cuando el ECO tenía la misma forma y color que el estímulo de muestra, (2) semejanza en forma o color, cuando el ECO tenía la misma forma pero diferente color o el mismo color pero diferente forma que el estímulo de muestra, respectivamente y (3) diferencia, cuando el ECO tenía una forma y un color distinto que el estímulo de muestra. El participante debía seleccionar con el ratón de la computadora el estímulo de comparación que creía correcto.

En la pantalla de descripciones se ofrecieron nueve opciones de texto para que el participante describiera lo que había hecho. Los textos se construyeron combinando los nombres de los tres estímulos de comparación y las tres relaciones posibles de igualación: identidad, semejanza y diferencia. La posición en que aparecía cada opción de texto cambió al azar en cada ensayo. El participante seleccionó su descripción al presionar el ratón de la computadora. 


\section{Pantalla 1 (Contexto de autoinstrucciones):}

\section{SELECCIONA UNA RESPUESTA}

En la siguiente pantalla aparecerá arriba un paralelogramo naranja y un trapecio lila, en medio un cuadrado verde limón, y abajo, un círculo amarillo, un rombo verde limón y un cuadrado verde limón ¿cuál de las figuras de abajo vas a seleccionar como respuesta correcta?

\begin{tabular}{|c|}
\hline el círculo amarillo \\
\hline el rombo verde limón \\
\hline \hline el cuadrado verde limón \\
\hline
\end{tabular}

Pantalla 2 (Contexto de igualación):

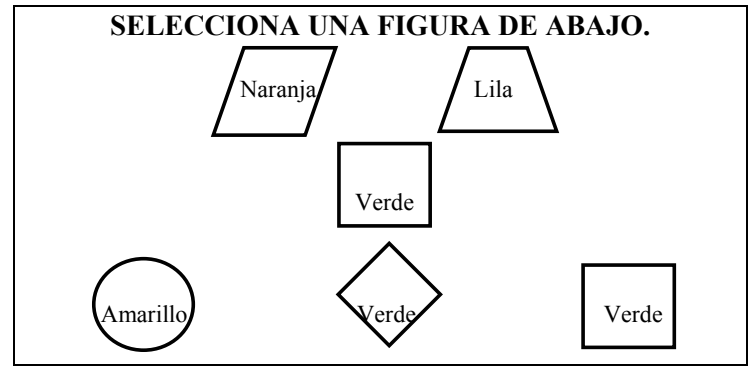

Pantalla 3 (Contexto de descripciones):

SELECCIONA LA OPCION QUE MEJOR DESCRIBA LO QUE HICISTE.

Seleccioné el círculo amarillo porque era idéntico a la figura del centro. Seleccioné el círculo amarillo porque era semejante a la figura del centro. Seleccioné el círculo amarillo porque era diferente a la figura del centro. Seleccioné el rombo verde limón porque era idéntico a la figura del centro. Seleccioné el rombo verde limón porque era semejante a la figura del centro. Seleccioné el rombo verde limón porque era diferente a la figura del centro. Seleccioné el cuadrado verde limón porque era idéntico a la figura del centro. Seleccioné el cuadrado verde limón porque era semejante a la figura del centro.

Seleccioné el cuadrado verde limón porque era diferente a la figura del centro.

Figura 1. Tarea de igualación de la muestra de segundo orden utilizada durante el entrenamiento de correspondencia y el entrenamiento con autoinstrucciones y descripciones. 
Los participantes trabajaron individualmente, aislados de ruido, en cubículos de $2 \mathrm{~m} \times 2 \mathrm{~m}$ que contaban con una computadora PC AT486 con monitor a color, teclado y ratón. La presentación de las figuras geométricas y la recolección de las respuestas de los participantes se realizaron por medio del paquete computacional ToolBook 1.5.

Al inicio de cada sesión de entrenamiento de correspondencia, se presentaron las siguientes instrucciones:

En esta parte del experimento se te presentarán 36 arreglos de figuras geométricas a los que denominamos "ensayos". En cada arreglo aparecen seis figuras: dos arriba, una en el centro y tres abajo. Debes escoger la figura de abajo que creas que va con la del centro tal y como se indica en las figuras de arriba. Sin embargo, tendrás que hacer algo ANTES y algo DESPUES de resolver cada ensayo.

ANTES de cada ensayo, se te describirá el arreglo de figuras con el que vas a trabajar. Se te pedirá que indiques cuál figura de abajo vas a seleccionar como respuesta correcta en dicha situación.

DESPUES de cada ensayo, se te presentarán una serie de opciones para elijas la que mejor describe lo que acabas de hacer.

La asignación de consecuencias dependió del tipo de entrenamiento de correspondencia. Para el entrenamiento en correspondencia decir-hacer (DH), la asignación de consecuencias ocurrió del siguiente modo. La respuesta de igualación (en la segunda pantalla) se retroalimentó con la palabra "acierto" si esa figura correspondía a la figura que había dicho que iba a escoger (en la primera pantalla), sin importar si dicha figura era la respuesta de igualación correcta o incorrecta. Se le dijo "error" cuando el participante no seleccionó el estímulo comparativo elegido en la pantalla de autoinstrucciones.

En el entrenamiento de correspondencia hacer-describir (HD), la retroalimentación se hizo sobre las descripciones de la ejecución (tercera pantalla). Al margen de que la respuesta de igualación fuera correcta o incorrecta, se le dijo "acierto" en la pantalla de descripciones cuando el participante describió correctamente lo que hizo en la igualación (segunda pantalla). Se le dijo "error" cuando no describió lo que había hecho. Dado que las opciones de texto para describir se hicieron combinando los nombres de los estímulos de comparación (ECO) y las tres relaciones posibles (identidad, semejanza y diferencia), se consideró que una descripción era correcta si mencionaba el ECO que había elegido y, además, utilizaba apropiadamente las palabras "idéntico", "semejante" o "diferente".

Durante el entrenamiento de correspondencia decir-describir (DD), se le dijo "acierto" en la pantalla de descripciones (tercera pantalla) cuando el participante mencionó el mismo estímulo de comparación que había elegido en 
la pantalla de autoinstrucciones (primera pantalla) y, además, utilizó apropiadamente las palabras "idéntico", "semejante" o "diferente".

Además de la retroalimentación continua de la correspondencia, al final de cada sesión de entrenamiento de correspondencia, apareció el total de aciertos que había acumulado el participante pero sin haberle aclarado que tales aciertos indicaban el número de veces que había sido consistente en hacer lo que dijo que iba a hacer, en describir lo que dijo que iba a hacer o en describir lo que había hecho, según el tipo de entrenamiento de correspondencia en cuestión. Es decir, la retroalimentación durante el entrenamiento de correspondencia no se refería a la precisión de la respuesta de igualación.

Entrenamiento con autoinstrucciones y descripciones.

El procedimiento fue similar al de entrenamiento en correspondencia ya que incluyó las mismas pantallas de autoinstrucciones, igualación y descripciones (ver figura 1). Las instrucciones que se dieron al inicio fueron iguales a las que se dieron en el entrenamiento de correspondencia. Sin embargo, la retroalimentación se refirió a la precisión de la respuesta de igualación. Así, se retroalimentó con la palabra "acierto" las elecciones correctas en la segunda pantalla y con la palabra "error" las respuestas incorrectas. Además de esta retroalimentación continua, al final de cada sesión apareció el número de aciertos totales.

\section{Entrenamiento instrumental}

Durante el entrenamiento instrumental sólo se utilizaron las pantallas de igualación (ver figura 1), sin autoinstrucciones ni descripciones. La mitad de los ensayos presentados requerían la selección del estímulo de comparación semejante y el resto debía igualarse por diferencia. Los ensayos se construyeron quasi-aleatoriamente para permitir que existiera un número igual de ensayos presentando la relación de semejanza en forma y la relación de semejanza en color. Además, permitió que la respuesta correcta se presentara igual número de veces en cada una de las posiciones de la hilera de abajo: derecha, centro e izquierda. Al inicio de cada sesión se dieron las siguientes instrucciones:

En la pantalla aparecerán seis figuras: dos arriba, una en el centro y tres abajo. Debes escoger la figura de abajo que crees que va con la del centro tal como se indica en las figuras de arriba. Para ello, coloca el cursor en la figura que elijas y presiona el botón izquierdo del ratón.

Las respuestas de igualación correctas se retroalimentaron con la palabra "acierto" y las incorrectas con la palabra "error". Sólo se retroalimentó positivamente las relaciones de semejanza o diferencia, pues la elección del ECO idéntico es una ejecución típica cuando no se dominan la discriminación condicional. Además de la retroalimentación continua, al final de cada sesión apareció el número de aciertos totales. 


\section{Preprueba y postprueba}

La preprueba fue similar al entrenamiento instrumental pero sin retroalimentar las elecciones del participante ni decirles al final el número de aciertos que habían obtenido. Los 36 ensayos presentaron arreglos de figuras geométricas seleccionados aleatoriamente de entre las sesiones experimentales de las cuatro fases de entrenamiento.

\section{Pruebas de transferencia intramodal}

Las pruebas de transferencia intramodal fueron similares al entrenamiento instrumental pero con colores y formas diferentes a los entrenados. Sólo se dio retroalimentación demorada ya que al final de la sesión se dijo al participante el número de aciertos totales que obtuvo.

\section{Pruebas de transferencia extramodal}

Las pruebas de transferencia extramodal fueron similares a las pruebas de transferencia intramodal pero cambiando la modalidad pertinente para la igualación. En lugar de la forma y el color, las relaciones de identidad, semejanza y diferencia se definieron a partir de la forma y el tamaño. Por ello, todos los estímulos fueron de color negro, variando el tamaño: grande o chico. De los 36 ensayos, 18 debían igualarse por diferencia (diferente forma y tamaño), 9 por semejanza en forma (misma forma y diferente tamaño) y 9 por semejanza en tamaño (mismo tamaño y diferente forma). Sólo se dio retroalimentación demorada en la forma de aciertos totales al final de la sesión.

\section{RESULTADOS}

En la figura 2 se presentan los porcentajes de aciertos para todos los participantes de los grupos experimentales en cada sesión del experimento. Las barras negras representan los resultados para las condiciones de pruebas: la preprueba en la primera sesión, la postprueba en la última sesión, y las pruebas de transferencia intramodal y extramodal (en ese orden) después de cada fase de entrenamiento. Se utilizan marcadores de texto diferenciados para los aciertos en cada pantalla del entrenamiento de correspondencia: rombos para el porcentaje de aciertos durante las autoinstrucciones, cuadrados para los aciertos en la pantalla de igualación y triángulos para el porcentaje de descripciones correctas. En la última fase de entrenamiento, sólo aparecen cuadrados ya que se corresponde al desempeño durante el entrenamiento instrumental. Los participantes se han ordenado de acuerdo a su ejecución durante la preprueba, de tal manera que, en la parte superior aparecen las gráficas para los participantes con $0 \%$ de aciertos durante la preprueba y en la parte inferior están las gráficas para los participantes con 


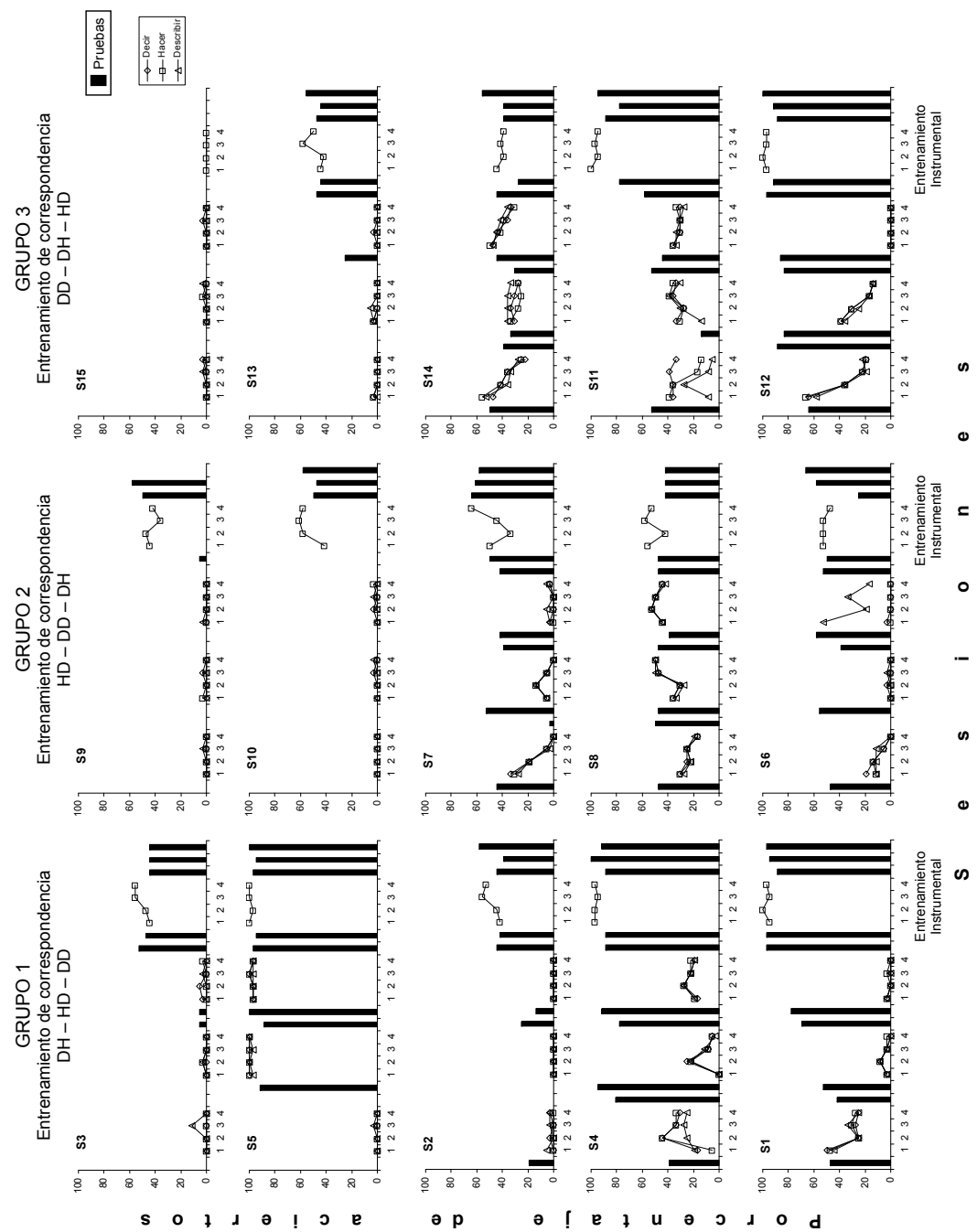

Figura 2. Porcentaje de aciertos en la igualación para cada una de las condiciones a las que fueron sometidos los participantes de los grupos experimentales. 
mayor número de aciertos durante la preprueba. Los participantes S5, S10 y S15 son los estudiantes de ingeniería.

Independientemente de la secuencia de entrenamiento de correspondencia, los participantes de los grupos experimentales con un desempeño menor a $30 \%$ de aciertos durante la preprueba (S3, S9, S15, S5, S10, S13 y S2) no lograron aprender la discriminación condicional con excepción del participante S5 quien aprendió la igualación durante la condición de prueba de transferencia extramodal después de la primera fase de entrenamiento. La sola retroalimentación demorada del desempeño correcto influyó para que S5, estudiante de ingeniería, aprendiera y siguiera utilizando los criterios de igualación correctamente. De los participantes de los grupos experimentales con más de $30 \%$ de aciertos durante la preprueba (S7, S14, S4, S8, S11, S1, S6 y S12), la mayoría de ellos tuvieron un desempeño diferenciado según los criterios con que se estableció la retroalimentación. Es decir, en las sesiones de entrenamiento de correspondencia (sin importar el tipo de correspondencia entrenada), el porcentaje de aciertos tendió a bajar (acercándose a 0 \%) mientras que, en las sesiones de prueba o entrenamiento instrumental, el porcentaje de aciertos tendió a subir (acercándose a $100 \%$ ). En un análisis detallado de sus ejecuciones, se pudo estimar que las elecciones con $0 \%$ de aciertos se debieron a que eligieron el ECO idéntico de manera exclusiva en todas las pantallas. Este comportamiento diferenciado fue más evidente en los participantes que sí lograron adquirir la discriminación condicional.

En la figura 3 se reportan los porcentajes de aciertos para los participantes de los grupos controles en cada condición experimental. Todos ellos lograron adquirir la discriminación condicional. Al comparar las figuras 2 y 3 , se puede observar que no hay diferencias entre los grupos controles con respecto a sus desempeños o la rapidez de adquisición de la igualación. Pero, sí hubo diferencias entre el grupo control 1 y los grupos experimentales con respecto al grado de correspondencia entre pantallas durante las tres primeras fases del experimento. Es decir, hubo mayor correspondencia para los participantes que se sometieron a algún tipo de entrenamiento de correspondencia (sin importar el tipo o la secuencia de entrenamiento de correspondencia) que los que se sometieron a la tarea en tres pantallas pero sin retroalimentación de correspondencia. Sólo hubo dos excepciones. El participante S6 sólo mostró correspondencia entre autoinstrucciones e igualación cuando fue sometido al entrenamiento de correspondencia decir-hacer. El participante S11 no presentó correspondencia entre pantallas al someterse al entrenamiento de correspondencia decir-describir en la fase 1. Al realizar un análisis cualitativo de su desempeño en esa fase de entrenamiento, se pudo establecer que dicho participante aprovechó la retroalimentación recibida para utilizar la etiqueta "idéntico" apropiadamente ya que eligió uno de los tres ECO's en las autoinstrucciones, eligió ese mismo ECO en la igualación pero al inicio 

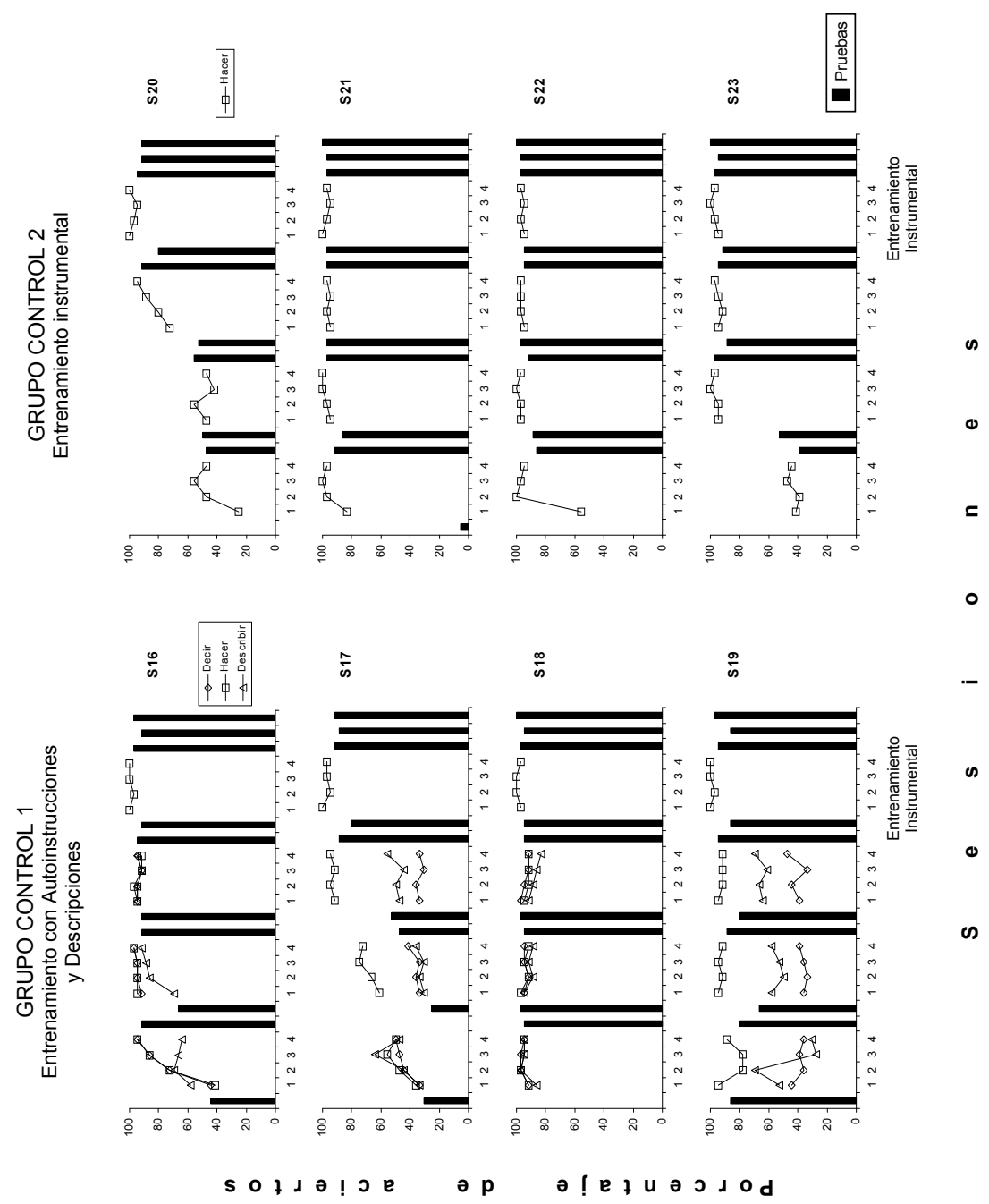

Figura 3. Porcentaje de aciertos en la igualación para cada una de las condiciones a las que fueron sometidos los participantes de los grupos controles. 
tomó cualquier descripción que mencionara la relación de identidad y al final hizo corresponder el ECO idéntico mencionado en la descripción con la frase "porque era idéntico a la figura del centro".

Por último, se llevó a cabo un análisis de varianza de un factor del promedio de porcentaje de respuestas correctas para cada grupo durante el entrenamiento instrumental ya que está condición se diseñó con el propósito de evaluar el efecto de las historias experimentales previas. El grupo experimental 1,2 y 3 tuvieron en promedio $78 \%, 50 \%$ y $57 \%$ de aciertos respectivamente, con una alta variabilidad entre los promedios de los participantes mientras que el grupo control 1 y 2 lograron $98 \%$ y $97 \%$ de aciertos respectivamente, con muy poca variabilidad entre los participantes. El estadístico $\mathrm{F}$ fue de 4.2257 (g.l. 4) con una probabilidad de 0.0138 lo que sugiere diferencias significativas entre los grupos. Sin embargo, no se encontraron diferencias significativas en el desempeño durante el entrenamiento instrumental cuando se compararon entre sí los grupos experimentales, cuando se compararon entre sí los grupos controles o cuando se compararon en base a la población estudiantil.

\section{DISCUSION}

En el presente estudio, se modificó una tarea de igualación de la muestra de segundo orden para someter a participantes de dos poblaciones estudiantiles distintas a diferentes historias de entrenamiento de correspondencia $\mathrm{DH}$, HD y DD. La tarea presentó ensayos divididos en tres pantallas; autoinstrucciones, igualación y descripciones. La retroalimentación sólo consideró la congruencia de las elecciones entre pantallas, por ejemplo, aunque el participante eligiera un estímulo de comparación incorrecto durante la igualación, se le informaba "acierto" si dicho ECO era el mismo que había elegido en la pantalla anterior de autoinstrucciones. Para describir su ejecución, el participante tenía que elegir un texto en donde se mencionara el ECO elegido y se completaba con la frase "porque era idéntico / semejante / diferente que la figura del centro". La retroalimentación exigió que el nombre de la relación mencionada (identidad, semejanza o diferencia) sí fuera ejemplificada con el ECO mencionado en el texto. Es decir, se promovió el uso correcto de las descripciones verbales de las relaciones entre el estímulo muestra y el ECO. La investigación tenía como propósito el análisis del impacto de este entrenamiento en la adquisición y generalidad de la discriminación condicional y, además, evaluar los patrones de correspondencias establecidos en cada tipo de entrenamiento.

El entrenamiento de correspondencia tuvo tres efectos sobre el desempeño de los participantes. Primero, fomentó el establecimiento de correspon- 
dencias entre las autoinstrucciones, la igualación y las descripciones. Este efecto fue independiente del tipo de correspondencia entrenada o de la secuencia de presentación. Segundo, se tuvieron desempeños diferenciados cuando la retroalimentación se refirió a la correspondencia que cuando la retroalimentación tomó en cuenta la respuesta de igualación correcta. Los participantes tendieron a igualar con el ECO idéntico durante el entrenamiento de correspondencia y a elegir el ECO correcto en las pruebas y el entrenamiento instrumental. Este desempeño diferenciado fue más notorio en participantes que obtuvieron en la preprueba un porcentaje de aciertos por arriba del nivel de azar. Tercero, hubo una interferencia con la adquisición de la igualación en los participantes con un bajo desempeño en la preprueba. Es decir, quienes no aprendieron la tarea de igualación bajo el entrenamiento de correspondencia, tampoco lo hicieron bajo el entrenamiento instrumental. El único participante con preprueba baja que aprendió la igualación fue un estudiante de ingeniería y lo hizo al someterse a la primera prueba de transferencia en donde recibió retroalimentación demorada. En estudios previos ya se ha encontrado que la retroalimentación demorada puede promover la adquisición de una igualación de segundo orden (Ribes y Castillo, 1998) pero, en este estudio, la sola retroalimentación demorada no fue suficiente para que los otros participantes aprendieran la tarea. ¿Cómo podemos considerar estos hallazgos en relación al papel funcional de las verbalizaciones (en forma de autoinstrucciones o descripciones de la ejecución) en el aprendizaje de una tarea de discriminación condicional como la igualación de la muestra de segundo orden?

Diversos autores han considerado que el desempeño en tareas de discriminación condicional puede ocurrir en dos niveles distintos (Baron y Galizio, 1990; Goldiamond, 1966; Ribes y López, 1985). Un nivel consiste en la adquisición de una respuesta a un estímulo con propiedades físicas específicas. Son ejecuciones situacionales que están influidas por las propiedades presentes y las relaciones inmediatas de los elementos de la tarea. Otro nivel, de orden superior, es la adquisición de una respuesta cuya dimensión de control revele una regla abstracta o un concepto como, por ejemplo, "similitud" o "diferencia" lo que supone una ejecución invariante ante propiedades de estímulos variantes (Cumming y Berryman, 1965; Goldiamond, 1966; Ribes, Moreno y Martínez, 1998). El dominio de la ejecución correcta parece ser condición necesaria pero no suficiente para un desempeño de orden superior que represente a una interacción extrasituacional o transituacional (Ribes y Martínez, 1990; Ribes y Rodríguez, 2001; Trigo, Martínez y Moreno, 1995). Cuando el participante es capaz de describir ejecuciones variadas como ejemplos de una misma regla de operación, su ejecución adquiere independencia de la situación particular (Lowenkron, 1989; Ribes y López, 1985; Ribes, Torres y Martínez, 1996, Vygotski, 1978). Una manera de evaluar experimentalmente 
la interacción extrasituacional o transituacional es midiendo la precisión de la ejecución en una prueba de transferencia extrarelacional o extradimensional (Varela y Quintana, 1995) en la que no se procura retroalimentación o se proporciona al final de la sesión (Ribes y Serrano, 2006)

Más recientemente, Ribes, Ontiveros, Torres, Calderón, Carvajal, Martínez y Vargas (2005) han argumentado que la discriminación condicional puede tener lugar de tres maneras funcionalmente distintas: a) perceptual, en donde el ECO correcto se determina por contraste entre los ECO's disponibles o por la saliencia relativa de alguna de las modalidades de los ECO's; b) verbal-modal, que podría corresponderse a una interacción extrasituacional, ya que involucra un reconocimiento verbal de las modalidades pertinentes en el criterio de igualación; y c) verbal-criterio, como interacción transituacional, que involucra el reconocimiento verbal directo del o los criterios de igualación que regulan la tarea. Para probar esta distinción, Serrano, García y López (2006) utilizaron una tarea de igualación de la muestra de segundo orden en donde usaron tres tipos de estímulos selectores: un par de figuras geométricas, un texto en donde se especificaba que había que elegir al que tuviera el mismo o diferente color y forma, y un texto con las palabras "identidad", "semejanza" y "diferencia". Estos autores encontraron que los participantes expuestos a estímulos selectores en forma de texto tuvieron una mejor ejecución en entrenamiento y en pruebas de transferencia que aquellos expuestos al par de figuras geométricas como estímulos selectores. Además, los participantes expuestos a textos que nombraban las relaciones tuvieron mejor desempeño en una prueba extradimensional que los participantes entrenados con textos que se referían a las modalidades de los estímulos.

A partir de estas delimitaciones conceptuales, podemos considerar que los participantes del presente estudio que se sometieron a un entrenamiento de correspondencia tendieron a contestar a la tarea de una manera perceptual aún en aquellos participantes con desempeños altos en pruebas. Dado que ésta es una interacción de menor complejidad que las interacciones verbal-modal o verbal-criterio, el entrenamiento de correspondencia no promovió la adquisición de la tarea de manera extrasituacional o transituacional e interfirió con la adquisición de la tarea en la mayoría de los participantes con preprueba baja. Esta tendencia a resolver la tarea en un nivel perceptual estuvo influida por los criterios con que se asignaron las consecuencias en el entrenamiento de correspondencia. Cuando la tarea involucró las pantallas de autoinstrucciones, igualación y descripciones pero con un criterio basado en la elección de la respuesta correcta, no se tuvo esta tendencia a resolverla en un nivel perceptual pero tampoco se promovió el establecimiento de correspondencia decir-hacer-describir. Es decir, el establecimiento de una correspondencia entre verbalizaciones de una ejecución y la ejecución no requiere de un comportamiento de orden superior que ejemplifique una 
ejecución orientada a metas o "regulación verbal". De manera similar, una ejecución de orden superior que ejemplifique la intervención funcional de criterios verbales no supone una correspondencia entre elementos verbales y no verbales de la tarea. Para evaluar esta premisa, será necesario diseñar futuros experimentos en donde se manipule ambos criterios de asignación de consecuencias: congruencia entre respuestas y precisión de las elecciones. Este rumbo en la investigación también fue sugerido por la evaluación más reciente del área de entrenamiento de correspondencia, realizada por Lloyd (2002) en donde argumenta que se debe partir del supuesto de que el establecimiento de correspondencias puede estar controlado por múltiples y diferentes contingencias.

\section{REFERENCIAS}

Baron, A. y Galizio, M. (1990). Control de la conducta operante humana por medio de instrucciones. En E. Ribes y P. Harzem (Eds.), Lenguaje y conducta (pp. 123167). México: Trillas.

Bentall, R. P., Lowe, C. F. y Beasty, A. (1985). The role of verbal behavior in human learning: II. Developmental differences. Journal of the Experimental Analysis of Behavior, 43, 165-181.

Bevill, A. R., Gast, D. L., Maguire, A. M. y Vail, C. O. (2001). Increasing engagement of preschoolers with disabilities through correspondence training and picture cues. Journal of Early Intervention, 24 (2), 129-145.

Birch, D. (1966). Verbal control of nonverbal behavior. Journal of Experimental Child Psychology, 4, 266-275.

Brodsky, G. (1967). The relation between verbal and non-verbal behavior change. Behavior Research \& Therapy, 5, 183-191.

Cumming, W. W. y Berryman, R. (1965). The complex discriminated operant studies of matching to sample and related problems. En D. I. Mostofsky (Ed.): Stimulus generalization, (pp. 284-330). Stanford: Stanford University Press.

Fujita, K. (1983). Acquisition and transfer of a high-order conditional discrimination performance in Japanese monkey. Japanese Psychological Research, 53, 1-18.

Goldiamond, I. (1966). Perception, language and conceptualization rules. En B. Kleinmuntz (Ed.), Problem solving: Research, method and theory (pp. 183-224). New York: Wiley.

Herruzo, J. y Luciano, C. (1994). Procedimientos para establecer la "correspondencia decir-hacer". Un análisis de sus elementos y problemas pendientes. Acta Comportamentalia, 2, 192-218.

Higa, W. R., Tharp, R. G. y Calkins, R. P. (1978). Developmental verbal control of behavior: Implications for self-instructional training. Journal of Experimental Child Psychology, 26, 489-497.

Israel, A. C. (1978). Some thoughts on correspondence between saying and doing. Journal of Applied Behavior Analysis, 11, 271-276. 
Israel, A. C. y Brown, M. S. (1977). Correspondence training, prior verbal training, and control of nonverbal behavior via control of verbal behavior. Journal of Applied Behavior Analysis, 10, 333-338.

Israel, A. C. y O'Leary, K. (1973). Developing correspondence between children's words and deeds. Child Development, 44, 577-581.

Lattal, K. E. y Doepke, K. J. (2001). Correspondence as conditional stimulus control: Insights from experiments with pigeons. Journal of Applied Behavior Analysis, 34, $127-144$

Lovaas, O. I. (1961). Interaction between verbal and nonverbal behavior. Child Development, 32, 329-336.

Lloyd, K. (2002). A review of correspondence training. Suggestions for a revival. Behavior Analyst, 25, 57-73.

Lowenkron, B. (1989). Instructional control of generalized relational matching to sample in children. Journal of the Experimental Analysis of Behavior, 52, 293-309.

Luciano, M. C., Herruzo, J. y Barnes-Holmes, D. (2001). Generalization of say-do correspondence. The Psychological Record, 51, 111-130.

Luciano, M. C., Molina-Cobos, F. J. y Gómez-Becerra, I. (2000). Say-do-report training to change chronic behaviors in mentally retarded subjects. Research in Developmental Disabilities, 21(5), 355-366.

Luria, A. R. (1964). The development of the regulatory role of speech. En R. J. C. Harper, C. C. Anderson, C. M. Christensen y S. M. Hunka (Eds.), The cognitive processes readings (pp. 601-622). United States of America: Prentince-Hall, Inc.

Meacham, J. A. (1979). The role of verbal activity in remembering the goals of actions. En G. Zivin (Ed.), The development of self-regulation through private speech (pp. 237-263). New York: John Wiley \& Sons.

Meichenbaum, D. y Goodman, J. (1969). The developmental control of operant motor responding by verbal operants. Journal of Experimental Child Psychology, 7, 553-565.

Morrison, R. S., Sainato, D. M., Benchaaban, D. y Endo, S. (2002). Increasing play skills of children with autism using activity schedules and correspondence training. Journal of Early Intervention, 25(1), 58-72.

Olsen-Woods, L. A., Miltenberger, R. G. y Foreman, G. (1998). Effects of correspondence training in an abduction prevention training program. Program, Child \& Family, 20, 15-34.

Paniagua, F. (1978). Efectos de las conductas intermedias sobre la correspondencia entre conducta verbal y no verbal. En Speller, P. (Ed.). Análisis de la Conducta. México: Trillas.

Paniagua, F. (1990). A procedural analysis of correspondence training techniques. The Behavior Analyst, 13, 107-119.

Paniagua, F. (2004). Utility of verbal-nonverbal correspondence training techniques in outpatient pediatric settings. Psychological Reports, 94(1), 317-326.

Paniagua, F. y Baer, D. (1982). The analysis of correspondence training as a chain reinforceable at any point. Child Development, 53, 786-798.

Paniagua, F. y Baer, D. (1988). Luria's regulatory concept and its misplacement in verbal-nonverbal correspondence training. Psychological Reports, 62, 371-378. 
Ralph, A., Hogan, S. J., Hill, M., Perkins, E., Ryan, J., Strong, L. (1998). Improving adolescent social competence in peer interactions using correspondence training. Education and Treatment of Children, 21(2), 171-194.

Ribes, E. (1990). Psicología General. México: Trillas.

Ribes, E. y Castillo, A. (1998). Interacción del tipo de entrenamiento y el tipo de respuesta de igualación en trasferencia en una discriminación condicional de segundo orden. Acta Comportamentalia, 6, 5-20.

Ribes, E. y López, F. (1985). Teoría de la conducta. Un análisis de campo y paramétrico. México: Trillas.

Ribes, E. y Martínez, H. (1990). Interaction of contingencies and rule instructions in the performance of human subjects in conditional discrimination. The Psychological Record, 40, 565-586.

Ribes, E., Moreno, D. y Martínez, C. (1998). Second-order discrimination in humans: The roles of explicit instructions and constructed verbal responding. Behavioural Processes, 42, 1-18.

Ribes, E., Ontiveros, S., Torres, C., Calderón, G., Carvajal, J., Martinez, C. y Vargas, I. (2005). La igualación de la muestra como selección de los estímulos de segundo orden: Efectos de dos procedimientos. Revista Mexicana de Análisis de la Conducta, 31, 1-22.

Ribes, E. y Rodríguez, M. E. (2001). Correspondence between instructions, performance, and self-descriptions in a conditional discrimination task: The effects of feedback and type of matching response. The Psychological Record, 51, 309333.

Ribes, E. y Serrano, M. (2006). "Efectos de tres tipos de preentrenamiento en la adquisición y transferencia de una tarea de igualación de la muestra", Acta Comportamentalia, 14(2), 145-169.

Ribes, E., Torres, C., Barrera, A. y Ramírez, L. (1995). Efectos de la variación modal de los estímulos en la adquisición y transferencia de una discriminación condicional en humanos adultos. Acta Comportamentalia, 3, 115-151.

Ribes, E., Torres, C. y Ramírez, L. (1996). Efecto de los modos de descripción en la adquisición y transferencia de una discriminación condicional de segundo orden en humanos adultos. Acta Comportamentalia, 4, 159-179.

Risley, T. R. y Hart, B. (1968). Developing correspondence between the non-verbal and verbal behavior of preschool children. Journal of Applied Behavior Analysis, 1, 267-281.

Rodríguez, M. E. (2000). Efecto del entrenamiento de la correspondencia decir-hacer, decir-describir y hacer-describir sobre la adquisición, generalidad y mantenimiento de una tarea de discriminación condicional en humanos. Acta Comportamentalia, 8, 41-75.

Rodríguez, M. E. (2002). Análisis de las variables que influyen en el establecimiento de correspondencias entre factores verbales y no verbales en tareas de discriminación condicional, tesis para obtener el grado de doctorado en ciencias del comportamiento, Universidad de Guadalajara.

Rogers-Warren, A. y Baer, D. M. (1976). Correspondence between saying and doing: teaching children to share and praise. Journal of Applied Behavior Analysis, 9, 335-354. 
Serrano, M., García, G. y López, A. (2006). Textos descriptivos de contingencia como estímulos selectores en igualación de la muestra con humanos. Acta Comportamentalia, 14(2), 131-143.

Stokes, J. V., Cameron, M. J., Dorsey, M. F. y Fleming, E. (2004). Task analysis, correspondence training, and general case instruction for teaching personal hygiene skills. Behavioral Interventions, 19 (2), 121-135.

Trigo, E., Martínez, R. y Moreno, R. (1995). Rule performance and generalization in a matching-to-sample task. The Psychological Record, 45, 223-240.

Varela, J. y Quintana, C. (1995). Comportamiento inteligente y su transferencia. Revista Mexicana de Análisis de la Conducta, 21, 47-66.

Vygotski, L. S. (1978). El desarrollo de los procesos psicológicos superiores. (Trad. al cast. 1979) España: Crítica. (Trabajo publicado a partir de escritos inéditos)

Whitman, T. L., Scibak, J. W., Butler, K. M., Ritcher, R. y Johnson, M. R. (1982). Improving class room behavior in mentally retarded children through correspondence training. Journal of Applied Behavior Analysis, 15, 545-564. 